\title{
Protecting Australia from Future Polio Outbreaks
}

\author{
Beverley J. Paterson*1, Nicolee Martin² and David N. Durrheim ${ }^{1}$ \\ ${ }^{1}$ Hunter Medical Research Unit, University of Newcastle, Newcastle, NSW, Australia; ${ }^{2}$ Australian Government Department of Health \\ and Ageing, Canberra, ACT, Australia
}

\section{Objective}

Few countries have tested the sensitivity of their polio surveillance systems, undertaken a comprehensive risk assessment or questioned whether existing polio surveillance strategies are the optimal surveillance at this stage of the global eradication initiative. To address this issue, a risk assessment and review of Australia's polio surveillance systems were undertaken to assess the potential risk of polio introduction by an infected person, product or specimen.

\section{Introduction}

The occurrence of global polio is at its lowest level, yet the goal of eradication remains elusive with the ongoing circulation in the three remaining endemic countries. Developed countries have a low index of suspicion for polio - relying on astute clinicians to detect imported cases and high immunisation rates to prevent community outbreaks. Australia, like all polio-free countries, remains at risk of a polio importation until polio is eradicated globally. Gaps in routine immunisation coverage coupled with weaknesses in surveillance may put developed countries, such as Australia, at risk of high impact polio outbreaks.

\section{Methods}

Methods utilised in the surveillance review and risk assessment included document review and semi-structured key informant interviews. Data were sourced from various published and unpublished government reports including immunisation coverage, movements of travellers, population immunity, population settlement statistics and polio surveillance data. Interviews were recorded, transcribed and thematically analysed.

\section{Results}

Vaccination coverage for polio in Australia is generally high but varies by geographical area, age group and Indigenous status. Lower areas of coverage have been identified among the general population. The potential for importation of a wild poliovirus exists in Australia with arrivals of travellers from polio endemic or re-infected countries, however serosurvey results indicate that there is sufficient herd immunity to prevent outbreaks of type 1 and type 2 poliovirus.

Australia meets some but not all of its polio surveillance objectives and there continues to be room for improvement, particularly stool sample surveillance targets. Case detection and outbreak mitigation were considered key reasons to undertake polio surveillance. Identified issues included: importance of supplemental environmental and enterovirus surveillance systems; potential high outbreak response costs resulting from an importation; continued importation risks; low stool sample collection rates; possible biosecurity inadequacy for imported samples and laboratory storage of biological samples containing poliovirus; and opportunities for improved legislative controls around polio. With most of the arrivals from polio endemic or re-infected countries settling in major cities, supplemental environmental surveillance in at least one major city would be useful.

\section{Conclusions}

Results from this work demonstrate that, while Australia has an impressive polio surveillance system, there are some gaps or risks that needed to be addressed. While the threat of an importation is low, ensuring that travellers and immigrants from areas where polio continues to occur are vaccinated against polio will help to address the risk. Polio vaccination coverage in Australia is high but there continue to be pockets where immunisation coverage is less than ideal, putting families at risk if an imported case were to be introduced into their area.

Recommendations from this work have resulted in improvements to surveillance, changes in biosecurity requirements for product importations that may contain wild poliovirus, and amendments to vaccination policies; all helping to protect Australia from future polio outbreaks.

\section{Keywords}

Surveillance; Polio; Needs assessment; Australia

\section{Acknowledgments}

The authors would like to acknowledge Dr Bruce Thorley and the team at the National Enterovirus Reference Laboratory at the Victorian Infectious Diseases Reference Laboratory.

*Beverley J. Paterson

E-mail: beverley.paterson@hnehealth.nsw.gov.au 